\title{
Optogenetic induction of appetitive and aversive taste memories in Drosophila
}

\author{
Meghan Jelen ${ }^{1}$, Pierre-Yves Musso ${ }^{1}$, Pierre Junca ${ }^{1}$, Michael D. Gordon $^{1 *}$ \\ ${ }^{1}$ Department of Zoology and Life Sciences Institute, University of British Columbia, Vancouver, \\ Canada \\ *Lead contact: gordon@zoology.ubc.ca
}

\section{SUMMARY}

Tastes are typically thought to evoke innate appetitive or aversive behaviours, prompting food acceptance or rejection. However, research in Drosophila melanogaster indicates that taste responses can be modified through experience-dependent changes in mushroom body circuits. In this study, we develop a novel taste learning paradigm using closed-loop optogenetics. We find that appetitive and aversive taste memories can be formed by pairing gustatory stimuli with optogenetic activation of sensory or dopaminergic neurons associated with reward or punishment. As with olfactory memories, distinct dopaminergic subpopulations drive the parallel formation of short- and long-term appetitive memories. Long-term memories are protein synthesis-dependent and have energetic requirements that are satisfied by a variety of caloric food sources or by direct stimulation of MB-MP1 dopaminergic neurons. Our paradigm affords new opportunities to probe plasticity mechanisms within the taste system and understand the extent to which taste responses are experience dependent. 


\section{INTRODUCTION}

Food selection is influenced by a complex set of factors including external sensory input, interoceptive circuits signaling internal state, and plasticity driven by past feeding experiences. The gustatory system plays a critical role in evaluating the nutritional qualities of foods, and is generally thought to evoke innate appetitive or aversive behavioural responses. However, the degree to which taste processing can be modified by learning is unclear.

In flies, taste detection is mediated by gustatory receptor neurons (GRNs) located on the proboscis, pharynx, legs, wing margins, and ovipositor ${ }^{1}$. GRNs express a range of chemosensory receptors for detection of sugars, bitters, salts, and other contact chemosensory cues ${ }^{2-4}$. GRNs project to the subesophageal zone (SEZ) of the fly brain where taste information is segregated based on modality, valence, and organ of detection ${ }^{5}$.

Although the valence of a specific taste is generally set, the intensity of the response can vary according to a variety of factors, including internal state. For example, starvation increases a fly's sensitivity towards sweet tastes, while blunting responses towards bitters. These starvationdependent changes occur through direct modulation of GRN activity ${ }^{6-9}$. Moreover, flies lacking essential nutrients such as amino acids and salts exhibit increased nutrient-specific preference towards protein and salt-rich foods ${ }^{10-12}$.

In addition to internal state-dependent changes in nutrient drive, fly taste responses can be altered by experience. Most notably, short-term taste-specific suppression of appetitive responses can be achieved through pairing with either bitter taste or noxious heat ${ }^{13-16}$. This plasticity requires an integrative memory association area called the mushroom body (MB), which is known to represent stimuli of different modalities, including taste ${ }^{13-15}$. Thus, while taste responses are carried out by innate circuits, they also exhibit experience-dependent changes driven by the adaptable networks of the MB ${ }^{14,17,18}$.

The MB is composed of approximately 4,000 intrinsic Kenyon cells (KCs), whose dendrites receive inputs from different sensory systems ${ }^{14,19-21}$. KCs form en passant synapses with mushroom body output neurons (MBONs), and MBONS send projections to neuropils outside of the MB to modulate behavioural output ${ }^{20,22}$. Connections between KCs and MBONs are depressed during learning, which skews the MB network towards approach or avoidance behaviours ${ }^{23-25}$. Reinforcement signals responsible for synaptic depression are conveyed by heterogenous dopaminergic neuron (DAN) populations when they are activated in coincidence with incoming sensory stimuli ${ }^{26,27}$. DANs are activated in response to rewarding and punishing stimuli within a fly's environment such as sucrose, bitter compounds, or shocks ${ }^{28,29}$. 
Interestingly, direct activation of DANs can function as an unconditioned stimulus (US) in some Drosophila associative learning paradigms ${ }^{17,30-32}$. Optogenetic or thermogenetic activation of the protocerebral anterior medial (PAMs) neurons, the rewarding DAN subpopulation, following or in coincidence with an odor conditioned stimulus (CS) results in the formation of an appetitive memory, while activation of punishing PPL1 DANs leads to the formation of an aversive memory ${ }^{15,33}$. Similarly, recent work on mammalian Pavlovian cue-reward learning shows that phasic optogenetic activation of specific dopaminergic subsets in the absence of a physical reward can lead to the formation of conditioned behaviours in mice ${ }^{34}$.

Appetitive short- and long-term memories (STM; LTM) are formed by independent PAM subpopulations, and the nutritional value of the sugar reward and satiation state of the fly contribute to the strength of the association ${ }^{17,28,33,35}$. Whereas STM may be formed under more flexible conditions with a sweet tasting reward, the formation of LTM requires a nutritious sugar. For example, L-glucose, a non-caloric, sweet tasting sugar can be used as a reward for the formation of STM, but only promotes LTM if paired with an energy source such as sorbitol $28,35,36$. Caloric sugars gate memory consolidation by promoting sustained rhythmic activity of MB-MP1 DANs ${ }^{35,37}$. Therefore, nutrient-sensing, internal state, and the gustatory system all play pivotal roles in reward learning ${ }^{38,39}$.

Although flies are known to exhibit aversive short-term taste memories, where appetitive taste responses are diminished through punishment ${ }^{14,15,40,41}$, the full extent to which taste behaviours are modifiable by learning is unknown. For example, can taste responses be enhanced by appetitive conditioning? Can flies form long-term memories about taste? These are difficult questions to answer using traditional methods for several reasons. First, appetitive association paradigms generally rely on food as the US, which could interfere with the representation of a taste CS and also modify future taste behaviours through changes in satiety state. Second, repeated temporal pairing of a taste CS with a US is difficult to achieve in flies without immobilization, making long-lasting memories difficult to test. To circumvent these issues, we developed an optogenetic learning paradigm that facilitates rapid, repeated CS/US pairing while maintaining similar satiation states between groups. In this paradigm, we couple a taste (the CS) with optogenetic GRN or dopaminergic neuron stimulation (the US) in order to study conditioned taste responses or 'taste memories'. For the purpose of our study, we will define a taste memory as a measurable change in a fly's behavioural response to a previously encountered taste stimulus.

Using our novel paradigm, we show that flies are capable of forming both appetitive and aversive short- and long-term taste memories. As in olfaction, appetitive taste memories are driven by discrete PAM populations, and activation of a single PAM subpopulation is sufficient to induce appetitive LTM. The formation of appetitive LTM requires de novo protein synthesis and is contingent on caloric intake. Moreover, sugar, certain amino acids, and lactic acid can 
provide the energy required to support LTM formation, and this requirement is also satisfied by thermogenetic activation of MB-MP1 neurons.

\section{RESULTS}

\section{Pairing GRN activation with a food source leads to taste memory formation}

We previously developed a system called the sip-triggered optogenetic behavioural enclosure (STROBE), in which individual flies are placed in an arena with free access to two food sources

${ }^{42}$. Interactions (mostly sips) with one of those foods triggers nearly instantaneous activation of a red LED, which can be used for optogenetic stimulation of neurons expressing CsChrimson. We reasoned that, if sipping on a tastant (the $\mathrm{CS}^{+}$) triggers activation of neurons that provide either positive or negative reinforcement, we may observe a change in the number of interactions a fly initiates upon subsequent exposure to the same $\mathrm{CS}^{+}$(Figure 1A).

We began by testing the efficacy of the STROBE in inducing aversive and appetitive memories through optogenetic activation of bitter and sweet GRNs, respectively (Figure 1B). Bitter GRN stimulation is known to activate PPL1 DANs, while sweet GRNs activate PAMs ${ }^{13,15,32,43}$. Moreover, bitter or sweet GRN activation with Gr66a- or Gr43a-Gal4 is sufficient for STM induction in taste and olfactory associative learning paradigms ${ }^{13,33}$. Therefore, we tested whether pairing GRN activation with feeding on a single taste modality could create an associative taste memory that altered subsequent behaviour to the taste.

In the aversive taste memory paradigm, interactions with $25 \mathrm{mM}$ sucrose $\left(\mathrm{CS}^{+}\right)$during training triggered LED activation of bitter neurons expressing CsChrimson, which led to $\mathrm{CS}^{+}$avoidance relative to plain agar (CS-) (Figure 1C). During testing, we disable the STROBE lights and measured preference towards $25 \mathrm{mM}$ sucrose $\left(\mathrm{CS}^{+}\right)$relative to agar $\left(\mathrm{CS}^{-}\right)$to see if flies have formed aversive taste memories. Indeed, ten minutes after training, flies that experienced bitter GRN activation during training showed a decreased sugar preference compared to control flies of the same genotype that were not fed the obligate CsChrimson cofactor all-trans-retinal, and retinal-fed control genotypes carrying either the Gal4 or UAS alone (Figure 1C). The same effect was produced by activation of PPK23 $3^{\text {glut } ~ ' h i g h ~ s a l t ' ~ G R N s, ~ w h i c h ~ a l s o ~ c a r r i e s ~ a ~ n e g a t i v e ~ v a l e n c e ~}$ in salt-satiated flies (Figure S1A, B). Importantly, these effects are not due to heightened satiety in trained flies because training in this paradigm is associated with fewer food interactions than controls.

For the appetitive memory paradigm, we chose $75 \mathrm{mM} \mathrm{NaCl}$ as the $\mathrm{CS}^{+}$, since flies show neither strong attraction nor aversion to this concentration of salt ${ }^{44}$. Interactions with the $\mathrm{CS}^{+}$triggered optogenetic activation of sweet neurons, either with Gr43a-Gal4, which labels a subset of leg and pharyngeal sweet neurons in addition to fructose-sensitive neurons in the protocerebrum 
(Figure 1B) or Gr64f-Gal4, which labels most peripheral sweet GRNs (Figure S1A). In both cases, sweet GRN activation produced increased preference for the $\mathrm{CS}^{+}$during training and testing 10-minutes later (Figure 1D and Figure S1C). Like the aversive memory paradigm, these effects cannot easily be explained through changes in internal state, since trained flies interacted more with the food during training and therefore should have a lower salt drive during testing. Interestingly, refeeding flies with standard medium directly after training in the appetitive paradigm led to a long-lasting preference for the $\mathrm{CS}^{+}$, revealed by testing 24-hours later (Figure $1 \mathrm{E}$ and Figure S1D). This stands in contrast to the aversive paradigm, where reduced preference for sugar following bitter GRN activation was absent during testing after 24 hours (Figure S1E).

\section{DAN activation is sufficient for the induction of short and long-lasting taste memories}

We next asked whether activating DANs during feeding could drive the formation of taste memories. Aversive short-term taste memory depends on multiple PPL1 DANs, including PPL1$\alpha ’ 2 \alpha 2$ and PPL1- $\alpha 3^{15}$, while appetitive short-term taste memories have not been previously reported. We first tested whether activating PPL1 DANs coincident with tastant interactions would lead to STM formation in the STROBE. Stimulation of PPL1 neurons reduced sucrose preference during training, and a reduced preference was also observed during short-term memory testing 10 minutes later (Figure 2A). Interestingly, unlike activation of bitter sensory neurons, PPL1 activation also produced a long-lasting aversive memory that was expressed 24 hours after training (Figure 2B).

To test the effect of appetitive DAN activation, we used flies expressing CsChrimson in PAM neurons under control of R58E02-Gal4. Intriguingly, although optogenetic activation of PAM neurons signals reward to the $\mathrm{MB}$, it did not affect preference towards light-paired $75 \mathrm{mM} \mathrm{NaCl}$ $\left(\mathrm{CS}^{+}\right.$) during training (Figure 2C, D). Nonetheless, this pairing resulted in appetitive memory expression during testing 10 minutes and 24 hours after training (Figure 2C, D). Thus, optogenetic activation of PAM neurons in the STROBE was able to write both short- and longlasting appetitive taste memories in the absence of acute effects on feeding. Importantly, appetitive memories were specific for the $\mathrm{CS}^{+}$, as flies trained with $\mathrm{NaCl}$ as the $\mathrm{CS}^{+}$did not exhibit elevated preference for the equally appetitive novel tastant monopotassium glutamate (MPG; Figure S2A)

Consistent with long-term olfactory memories induced by DAN activation, we found longlasting taste memory consolidation required an energetic food source, and therefore flies were refed for a brief period after training. Flies fed 7 hours post-training, after the memory consolidation time period defined in olfactory memory, did not express taste memories during testing (Figure 2D) ${ }^{35}$. Thus, the contingencies governing the formation and expression of taste memories in Drosophila seem to be similar to those previously discovered for olfaction. 


\section{The MB is required for the formation of short- and long-lasting taste memories}

Prior research indicates that the intrinsic neurons of the $\mathrm{MB}$ are required for aversive taste memory formation ${ }^{15}$. To confirm that the MB is required for appetitive taste memory formation, we silenced this neuropil throughout our long-lasting memory assay using tetanus toxin expressed under control of the pan-MB driver R13F02-LexA. After pairing Gr43a activation with $\mathrm{NaCl}$ feeding, flies with silenced $\mathrm{MBs}$ did not exhibit elevated preference for salt during either the short-term or long-lasting taste memory assays (Figure 3A, B). Similarly, PAM activation during feeding led to a sustained increase in preference for the $\mathrm{NaCl}$ tastant in control groups for both the STM and long-lasting memory assay, but not in flies with silenced MBs (Figure 3C, D). These findings indicate that MB intrinsic neurons play a pivotal role in the formation of appetitive taste memory (Figure $3 \mathrm{~F}$ ).

To assess whether the molecular underpinnings of 24-hour appetitive taste memory are consistent with classic olfactory LTM - which requires de novo protein synthesis during memory consolidation - we fed flies all-trans-retinal laced with the protein synthesis inhibitor cycloheximide $(\mathrm{CXM}){ }^{17}$. As expected, flies fed CXM prior to training were unable to form longterm taste memories, in contrast to vehicle controls (Figure 3E). These results confirm that protein synthesis is necessary for long-term taste memory formation (Figure 3F).

\section{Distinct PAM subpopulations induce appetitive short- and long-term taste memories}

Distinct, non-overlapping subpopulations of PAM neurons, labeled by R48B04-Gal4 and R15A04-Gal4, mediate the formation of appetitive short- and long-term olfactory memories, respectively ${ }^{33}$. Moreover, it has been hypothesized that two differential reinforcing effects of sugar reward - sweet taste and nutrition, are encoded by these segregated STM and LTM neural populations ${ }^{33}$. We tested both populations in our appetitive STROBE memory assays to determine if the activation of these separate PAM clusters would support the formation of parallel short- and long-term taste memories. R48B04>CsChrimson flies formed appetitive short-term but not long-term taste memories, as shown by the higher salt preference of flies expressing active CsChrimson during STM testing but not LTM testing (Figure 4A, B). Conversely, activation of R15A04-Gal4 neurons produced LTM but not STM (Figure 4C, D). These results indicate that, much like appetitive olfactory memory, short- and long-term taste memories are formed in parallel by discrete PAM sub-populations.

Next, we wondered whether activation of a single PAM cell subtype, PAM- $\alpha 1$, would be sufficient to induce taste memories. PAM- $\alpha 1$ neurons project to an MB compartment innervated by MBON- $\alpha 1$, which in turn feeds back onto PAM- $\alpha 1$ to form a recurrent reward loop necessary for the formation of appetitive olfactory LTM ${ }^{45,46}$. Consistent with its role in olfactory memory, activation of this PAM cell type in the STROBE with drivers MB043B-Gal4 or MB299B-Gal4 
was sufficient to drive appetitive long-term, but not short-term, taste memory formation (Figure 4E, F and Figure S3A, B).

Interestingly, activation of another PAM subset labelled by MB301B-Gal4 produced a higher preference for the salt CS during training, yet no sustained changes in taste preference during short- or long-term memory testing (Figure 4G, H). This demonstrates that the reward signaling associated with PAM cell activation occurs on multiple timescales to produce acute, short-, or long-term changes in behavior. Notably, the trend toward lower salt preference during testing in this experiment may reflect reduced salt drive due to increased salt consumption during training.

\section{Caloric food sources are required for the formation of associative long-term taste memories}

Because refeeding shortly after training is required for the consolidation of appetitive long-term taste memories, we next asked what types of nutrients would support this memory formation. As expected, refeeding with L-glucose, a non-caloric sugar, did not lead to the formation of associative long-term taste memories (Figure 5A, B). However, along with sucrose, refeeding with lactic acid, yeast extract, and L-alanine promoted long-term memory, while L-aspartic acid did not. These results indicate that in addition to sucrose, other caloric nutrients can provide sufficient energy for long-term taste memory formation. Moreover, 7-hour delayed refeeding of each nutrient failed to support memory formation (Figure 5B). Thus, similar to olfactory LTM, the formation of appetitive taste LTM is dependent on an energy source being readily available during the memory consolidation window ${ }^{35,38}$.

Our findings concerning the formation and expression of appetitive taste LTM bear striking similarities to those of olfactory LTM in terms of MB circuitry, dependence on protein synthesis, and energetic requirements. This led us to wonder if the energy gating performed by MB-MP1 neurons, which signal onto the mushroom body and promote energy flux in MB neurons during LTM, perform a similar function in taste memory ${ }^{35,47,48}$. To test this hypothesis, we activated MB-MP1 neurons directly after training using UAS-TRPAI and delayed refeeding to outside the memory consolidation window. Compared to genetic controls, flies in which MB-MP1 neurons were activated post training show significantly elevated memory scores during testing (Figure 6A, B). This confirms that MB-MP1 activation is sufficient to drive memory consolidation during long-term appetitive taste memory formation (Figure 6C).

\section{DISCUSSION}

Gustation plays a vital role in determining the suitability of foods for ingestion. Yet, little is known about how experience influences higher-order taste representations and contributes to the continuous refinement of food selection. In fact, in flies, a memory system for the recollection of appetitive taste CS has not been described. In this study, we use the STROBE to establish a 
novel learning paradigm and further investigate the formation and expression of taste memories. We demonstrate that flies are capable of forming short- and long-term appetitive and aversive taste memories towards two key nutrients - salt, and sugar. Much like olfactory memory, associative taste memory formation occurs within the MB and follows many of the same circuit and energetic principles.

Appetitive taste memory has been severely understudied in Drosophila due to several inherent experimental design issues. First, in other types of associative reward learning - such as olfactory and visual - sucrose is often used as the unconditioned stimulus (US) or reward, which can interact with the representation of a gustatory CS. For example, bitter and sweet tastes interact at both the receptor and circuit levels, and salt responses overlap with most other taste stimuli in the periphery ${ }^{11,49,50}$. Second, changes in internal state due to ingestion of specific nutrients during learning can either influence behavior independent of memory formation, or directly affect memory formation or expression. Most notably, if $\mathrm{CS}^{+}$and $\mathrm{CS}^{-}$groups ingest different amounts of a nutrient during training, it will likely influence the specific drive for the taste of that modality during testing ${ }^{12,51}$. Nevertheless, rats' hedonic response to bitter compounds can be made more positive through pairing with sugar, and human studies suggest that children's taste palates are malleable based on positive experiences with bitter vegetables ${ }^{52-}$ 55. Thus, despite the constraints of measuring taste memories in a controlled setting, appetitive taste plasticity is very likely an ethologically important process.

To circumvent the complexities associated with appetitive taste memory formation, we decided to directly stimulate DANs as the flies experienced taste inputs through feeding. This also afforded the opportunity to interrogate the roles of specific DAN populations. Taking a hypothesis driven approach, we confirmed that PAM neural subpopulations reinforce taste percepts much like olfactory inputs, and that short- and long-term memories are processed by distinct subpopulations. For example, activating $\beta$ '2, $\gamma 4$, and $\gamma 5$ compartments with $R 48 B 04$ Gal4 produces STM in both olfactory and taste paradigms, while activation of $\alpha 1, \beta$ ' $1, \beta 2$, and $\gamma 5$ with R15A04-Gal4 produces LTM in both. These results confirm that appetitive STM and LTM are processed in parallel in the MB ${ }^{33,56}$. Given that tastes, like odours, activate the KC calyces, we speculate that optogenetic stimulation of PAM neurons during feeding modulates the strength of KC-MBON synaptic connections ${ }^{14}$. Notably, activation of single PAM cell type produced different forms of memory in the STROBE. For example, stimulating PAM- $\alpha 1$ neurons during feeding drives appetitive taste LTM, while activation of PAM- $\beta$ ' 1 was immediately rewarding.

A unique aspect of our long-term taste learning paradigm is that we uncoupled the US from a caloric food source. By doing this we were able to probe the energetic constraints gating LTM formation. For years it has been reported that long-term memory formation in Drosophila requires the intake of caloric sugar. Here, we demonstrate that the caloric requirements of long- 
term memory formation can be quenched by food sources other than sucrose, such as lactic acid, and protein. Moreover, it seems that at least one amino-acid, L-alanine, is able to provide adequate energy, while L-aspartic acid cannot. We theorize that these foods may provide flies readily accessible energy, as neurons are able to metabolize both lactic acid and L-alanine into pyruvate to fuel the production of ATP via oxidative phosphorylation ${ }^{57}$.

Energy gating in the MB is thought to be regulated by the MB-MP1-DANs. MB-MP1 neuron oscillations activate increased mitochondrial energy flux within the $\mathrm{KCs}$, which is both necessary and sufficient to support LTM ${ }^{48}$. To demonstrate sufficiency in our assay, we activated MB-MP1 neurons with TRPA1 directly after fly training, which effectively substitutes for a caloric food source and allows LTM formation (Figure 6C). These results suggest that MB-MP1 neurons integrate energy signals during formation of multiple types of LTM and may be influenced by a variety of caloric foods.

Intriguingly, we observed both positive and negative taste memories following optogenetic GRN stimulation concurrent with taste detection. The activation of bitter GRNs paired with sucrose led to the formation of STM, which agrees with previous research demonstrating that thermogenetic stimulation of bitter GRNs can act as a negative US, and lead to taste learning in short-term paradigms ${ }^{13}$. Strikingly, bitter GRN activation was not sufficient for the formation of LTM in our assay. One possible explanation is that because bitter GRN activation strongly inhibits feeding, the number of pairings between sugar taste and bitter activation is insufficient to induce LTM. By contrast, PPL1 activation is less aversive during training and therefore allows more associations.

Unlike bitter GRNs, activation of sweet GRNs prompted both STM and LTM formation. This indicates that lasting changes in the value of specific tastes can occur in response to temporal association of different taste modalities, and raises the possibility that such plasticity plays an important role in animals' ongoing taste responses. Future experiments using the STROBE paradigm could further probe the molecular and circuit mechanisms underlying such changes and advance our understanding of how taste preferences may be shaped by experience over an animal's lifetime.

\section{MATERIALS AND METHODS}

\section{Fly strains}

Fly stocks were raised on a standard cornmeal diet at $25^{\circ} \mathrm{C}, 70 \%$ relative humidity. For neuronal activation 20XUAS-IVS-CsChrimson.mVenus (BDCS, stock number: 55135) was used. Dopaminergic PAM expression was targeted using previously described lines: R58E02-GAL4 ${ }^{35}$; R58E02-LexA, R48B04-GALA, R15A04-GALA, R13F02-LexA, and R30E11-LexA obtained from 
Bloomington (BDCS, stock numbers: 52740, 50347, 48671, 52460, 54209); and MB split-GAL4 lines MB043B-GAL4, MB504B-GAL4, MB299B-GAL4, MB301B-GAL4 from Janelia Farms ${ }^{23}$. GRN expression was driven using Gr43a-GAL4, Gr64f-GAL4 ${ }^{58}$, Gr66a-GAL4 ${ }^{59}$, and PPK23 $3^{\text {glut }}$ GAL4 (PPK23-GAL4, Gr66a-LexA::VP16, LexAop-Gal80 ${ }^{11}$ ). LexAop-tnt was previously described $^{60}$. For temperature activation experiments LexAop-TrpAl was used $^{32}$.

\section{STROBE experiments}

Mated female Drosophila were collected 2-3 days post eclosion and transferred into vials containing $1 \mathrm{ml}$ of standard cornmeal medium supplemented with $1 \mathrm{mM}$ all-trans-retinal (Sigma \#R2500) or an ethanol vehicle control. Flies were maintained on this diet for 2 days in a dark environment. 24 hours prior to experimentation flies were starved at $25^{\circ} \mathrm{C}, 70 \%$ relative humidity, on $1 \%$ agar supplemented with $1 \mathrm{mM}$ all-trans-retinal or ethanol vehicle control.

\section{STROBE training protocol}

During the training phase for the short-term memory experiments the STROBE was loaded with $4 \mu \mathrm{L}$ of tastant (salt: Sigma \#S7653 or sucrose: Sigma \#S7903) on channel 1 and $4 \mu \mathrm{L} 1 \%$ agar on channel 2 . The red LED was triggered only when a fly interacted with the tastant in channel 1. The duration of the training period was 40 minutes. For the STM training protocol, flies were then transferred to clean empty vials for 10 minutes while the experimental apparatus was cleaned. The training and testing phases of LTM experiments were performed as described for the STM experiments with the following exception: after the 40-minute training period flies, were transferred individually into vials containing standard cornmeal diet or nutrient of interest (sucrose: Sigma \#S7903, L-glucose: Sigma \#G5500, lactic acid: Sigma \#69785, yeast extract: Sigma \#Y1625, L-alanine: Sigma \#05129, L-aspartic acid: Sigma \#11230) and allowed to feed for 1 hour. They were then transferred into $1 \%$ agar starvation vials and kept at $18^{\circ} \mathrm{C}$ until the testing component of the experiment. For MB-MB1 activation experiments, after training flies were placed at $29^{\circ} \mathrm{C}, 70 \%$ relative humidity for 1 hour on $1 \%$ agar starvation vials. They were then transferred to $18^{\circ} \mathrm{C}$ and refed 8 hours later, outside of the memory consolidation. After 1 hour of feeding they were once again transferred into $1 \%$ agar starvation vials and kept at $18^{\circ} \mathrm{C}$ until the retrieval component of the experiment. The preference index for each individual fly was calculated as: (sips from channel 1 - sips from channel 2)/(sips from channel $1+$ sips from channel 2). All experiments were performed with a light intensity of $11.2 \mathrm{~mW} / \mathrm{cm}^{2}$ at $25^{\circ} \mathrm{C}, 70 \%$ relative humidity.

\section{STROBE testing protocol}

During testing, $4 \mu \mathrm{L}$ of the same tastant (salt: Sigma \#S7653, sucrose: Sigma \#S7903, MPG: Sigma \#G1501) was reloaded into channel one and $4 \mu \mathrm{L}$ of $1 \%$ agar on channel 2 . The optogenetic component of the system was deactivated such the red LED would no longer trigger if a fly interacted with the tastant. Flies were reloaded individually into the same arenas. The duration of the testing phase was 1 hour. The preference index for each individual fly was 
calculated as: (sips from channel 1 - sips from channel 2$) /($ sips from channel $1+$ sips from channel 2).

\section{Immunofluorescence microscopy}

Brain staining protocols were performed as previously described ${ }^{49}$. Briefly, brains were fixed for 1 hour in $4 \%$ paraformaldehyde and dissected in PBS $+0.1 \%$ TritonX. After dissection brains were blocked in 5\% NGS diluted with PBST for 1 hour. Brains were probed overnight at $4{ }^{\circ} \mathrm{C}$ using the following primary antibody dilutions: rabbit anti-GFP (1:1000, Invitrogen \#A11122), and mouse anti-brp (1:50, DSHB \#nc82). After a 1hour wash period secondary antibodies goat anti-rabbit Alexa-488 (1:200, Invitrogen \#A11008) and goat anti-mouse Alexa-568 (1:200, Invitrogen \#A11030) were applied and incubated for 1 hour at room temperature to detect primary antibody binding. Slowfade gold was used as an antifade mounting medium.

Slides were imaged under a 25x water immersion objective using a Leica SP5 II Confocal microscope. All images were taken sequentially with a z-stack step size at $1 \mu \mathrm{m}$, a line average of 2 , speed of $200 \mathrm{~Hz}$, and a resolution of 1024 x 1024 pixels. Image J was used to compile slices into a maximum intensity projection ${ }^{11}$.

\section{Statistical analysis}

All statistical analyses were executed using GraphPad Prism 6 software. Sample size and statistical tests performed are provided in the Figure legends. For Dunnett's post hoc analyses, the lowest statistically significant value is shown on top of the experimental group as compared to various controls. Replicates are biological replicates, using different individual flies from 2 or more crosses. Sample sizes were based on previous experiments in which effect size was determined. Data was excluded on the basis of STROBE technical malfunctions for individual flies and criteria for data exclusion are as follows: i) if the light system was not working during training for individual arenas ii) if during training or testing a fly did not meet a standard minimum \# of interactions for that genotype iii) if during training or testing the STROBE recorded an abnormally large \# of interactions for that genotype iiii) technical malfunctions due to high channel capacitance baseline activity v) if a fly was dead in an arena.

\section{DATA AVAILABILITY}

All data are available in the manuscript and Supplementary Information, or are available upon request from the corresponding author. 


\section{ACKNOWLEDGEMENTS}

We thank Celia Lau for the original SEZ diagram models, and members of the Gordon lab for comments on the manuscript. Funding was provided by Natural Sciences and Engineering Research Council (NSERC) (RGPIN-2016-03857 and RGPAS-49246-16) and a Michael Smith Foundation for Health Research Scholar Award (MDG)

\section{AUTHOR CONTRIBUTIONS}

M.J, P.Y.M, and M.D.G conceived the project. M.J and M.D.G wrote the manuscript. M.J ran all experiments. P.Y.M and P.J offered experimental advice. M.D.G supervised the project.

\section{DECLARATION OF INTERESTS}

The authors declare no competing interests.

\section{SUPPLEMENTAL INFORMATION}

Supplemental information includes 3 figures. 


\section{REFERENCES}

1. Stocker, R. F. The organization of the chemosensory system in Drosophila melanogaster: a rewiew. Cell Tissue Res. 275, 3-26 (1994).

2. Hiroi, M., Meunier, N., Marion-Poll, F. \& Tanimura, T. Two antagonistic gustatory receptor neurons responding to sweet-salty and bitter taste inDrosophila. J. Neurobiol. 61, 333-342 (2004).

3. Thorne, N., Chromey, C., Bray, S. \& Amrein, H. Taste Perception and Coding in Drosophila. Curr. Biol. 14, 1065-1079 (2004).

4. Zhang, Y. et al. Coding of Sweet, Bitter, and Umami Tastes: Different Receptor Cells Sharing Similar Signaling Pathways. 9 (2003).

5. Marella, S. et al. Imaging Taste Responses in the Fly Brain Reveals a Functional Map of Taste Category and Behavior. Neuron 49, 285-295 (2006).

6. Inagaki, H. K. et al. Visualizing Neuromodulation In Vivo: TANGO-Mapping of Dopamine Signaling Reveals Appetite Control of Sugar Sensing. Cell 148, 583-595 (2012).

7. Inagaki, H. K., Panse, K. M. \& Anderson, D. J. Independent, Reciprocal Neuromodulatory Control of Sweet and Bitter Taste Sensitivity during Starvation in Drosophila. Neuron 84, 806-820 (2014).

8. LeDue, E. E. et al. Starvation-Induced Depotentiation of Bitter Taste in Drosophila. Curr. Biol. 26, 2854-2861 (2016).

9. Marella, S., Mann, K. \& Scott, K. Dopaminergic Modulation of Sucrose Acceptance Behavior in Drosophila. Neuron 73, 941-950 (2012).

10. Corrales-Carvajal, V. M., Faisal, A. A. \& Ribeiro, C. Internal states drive nutrient homeostasis by modulating exploration-exploitation trade-off. eLife 5, e19920 (2016). 
11. Jaeger, A. H. et al. A complex peripheral code for salt taste in Drosophila. eLife 7, e37167 (2018).

12. Steck, K. et al. Internal amino acid state modulates yeast taste neurons to support protein homeostasis in Drosophila. 29 (2018).

13. Keene, A. C. \& Masek, P. Optogenetic induction of aversive taste memory. Neuroscience 222, 173-180 (2012).

14. Kirkhart, C. \& Scott, K. Gustatory Learning and Processing in the Drosophila Mushroom Bodies. J. Neurosci. 35, 5950-5958 (2015).

15. Masek, P., Worden, K., Aso, Y., Rubin, G. M. \& Keene, A. C. A Dopamine-Modulated Neural Circuit Regulating Aversive Taste Memory in Drosophila. Curr. Biol. 25, 1535-1541 (2015).

16. Tauber, J. M. et al. A subset of sweet-sensing neurons identified by IR56d are necessary and sufficient for fatty acid taste. PLOS Genet. 13, e1007059 (2017).

17. Colomb, J., Kaiser, L., Chabaud, M.-A. \& Preat, T. Parametric and genetic analysis of Drosophila appetitive long-term memory and sugar motivation. Genes Brain Behav. 8, 407415 (2009).

18. Krashes, M. J. et al. A Neural Circuit Mechanism Integrating Motivational State with Memory Expression in Drosophila. Cell 139, 416-427 (2009).

19. Schwaerzel, M. et al. Dopamine and Octopamine Differentiate between Aversive and Appetitive Olfactory Memories in Drosophila. J. Neurosci. 23, 10495-10502 (2003).

20. Tanaka, N. K., Tanimoto, H. \& Ito, K. Neuronal assemblies of the Drosophila mushroom body. J. Comp. Neurol. 508, 711-755 (2008). 
21. Vogt, K. et al. Shared mushroom body circuits underlie visual and olfactory memories in Drosophila. eLife 3, e02395 (2014).

22. Crittenden, J. R., Skoulakis, E. M. C., Han, K.-A., Kalderon, D. \& Davis, R. L. Tripartite Mushroom Body Architecture Revealed by Antigenic Markers. E R N N G 14 (1998).

23. Aso, Y. et al. Mushroom body output neurons encode valence and guide memory-based action selection in Drosophila. eLife 3, e04580 (2014).

24. Cohn, R., Morantte, I. \& Ruta, V. Coordinated and Compartmentalized Neuromodulation Shapes Sensory Processing in Drosophila. Cell 163, 1742-1755 (2015).

25. Perisse, E. et al. Different Kenyon Cell Populations Drive Learned Approach and Avoidance in Drosophila. Neuron 79, 945-956 (2013).

26. Gervasi, N., Tchénio, P. \& Preat, T. PKA Dynamics in a Drosophila Learning Center: Coincidence Detection by Rutabaga Adenylyl Cyclase and Spatial Regulation by Dunce Phosphodiesterase. Neuron 65, 516-529 (2010).

27. Tomchik, S. M. \& Davis, R. L. Dynamics of Learning-Related cAMP Signaling and Stimulus Integration in the Drosophila Olfactory Pathway. Neuron 64, 510-521 (2009).

28. Burke, C. J. \& Waddell, S. Remembering Nutrient Quality of Sugar in Drosophila. Curr. Biol. 21, 746-750 (2011).

29. Mao, Z. \& Davis, R. L. Eight different types of dopaminergic neurons innervate the Drosophila mushroom body neuropil: anatomical and physiological heterogeneity. Front. Neural Circuits 3, (2009).

30. Aso, Y. et al. Three Dopamine Pathways Induce Aversive Odor Memories with Different Stability. PLoS Genet. 8, e1002768 (2012). 
31. Claridge-Chang, A. et al. Writing Memories with Light-Addressable Reinforcement Circuitry. Cell 139, 405-415 (2009).

32. Liu, C. et al. A subset of dopamine neurons signals reward for odour memory in Drosophila. Nature 488, 512-516 (2012).

33. Yamagata, N. et al. Distinct dopamine neurons mediate reward signals for short- and longterm memories. Proc. Natl. Acad. Sci. 112, 578-583 (2015).

34. Saunders, B. T., Richard, J. M., Margolis, E. B. \& Janak, P. H. Dopamine neurons create Pavlovian conditioned stimuli with circuit-defined motivational properties. Nat. Neurosci. 21, 1072-1083 (2018).

35. Musso, P.-Y., Tchenio, P.\& Preat, T. Delayed Dopamine Signaling of Energy Level Builds Appetitive Long-Term Memory in Drosophila. Cell Rep. 10, 1023-1031 (2015).

36. Musso, P.-Y., Lampin-Saint-Amaux, A., Tchenio, P.\& Preat, T. Ingestion of artificial sweeteners leads to caloric frustration memory in Drosophila. Nat. Commun. 8, 1803 (2017).

37. Pavlowsky, A., Schor, J., Plaçais, P.-Y.\& Preat, T. A GABAergic Feedback Shapes Dopaminergic Input on the Drosophila Mushroom Body to Promote Appetitive Long-Term Memory. Curr. Biol. 28, 1783-1793.e4 (2018).

38. Fujita, M. \& Tanimura, T. Drosophila Evaluates and Learns the Nutritional Value of Sugars. Curr. Biol. 21, 751-755 (2011).

39. Huetteroth, W. et al. Sweet Taste and Nutrient Value Subdivide Rewarding Dopaminergic Neurons in Drosophila. Curr. Biol. 25, 751-758 (2015).

40. Kim, H., Kirkhart, C. \& Scott, K. Long-range projection neurons in the taste circuit of Drosophila. eLife 6, e23386 (2017). 
41. Masek, P. \& Scott, K. Limited taste discrimination in Drosophila. Proc. Natl. Acad.Sci.107, 14833-14838 (2010).

42. Musso, P.-Y. et al. Closed-loop optogenetic activation of peripheral or central neurons modulates feeding in freely moving Drosophila. eLife 8, e45636 (2019).

43. Miyamoto, T., Slone, J., Song, X. \& Amrein, H. A Fructose Receptor Functions as a Nutrient Sensor in the Drosophila Brain. Cell 151, 1113-1125 (2012).

44. Zhang, Y. V., Ni, J.\& Montell, C. The Molecular Basis for Attractive Salt-Taste Coding in Drosophila. Science 340, 1334-1338 (2013).

45. Aso, Y.\& Rubin, G. M. Dopaminergic neurons write and update memories with cell-typespecific rules. eLife 5, e16135 (2016).

46. Ichinose, T. et al. Reward signal in a recurrent circuit drives appetitive long-term memory formation. eLife 4, e10719 (2015).

47. Plaçais, P.-Y. et al. Slow oscillations in two pairs of dopaminergic neurons gate long-term memory formation in Drosophila. Nat. Neurosci. 15, 592-599 (2012).

48. Plaçais, P.-Y. et al. Upregulated energy metabolism in the Drosophila mushroom body is the trigger for long-term memory. Nat. Commun. 8, 15510 (2017).

49. Chu, B., Chui, V., Mann, K. \& Gordon, M. D. Presynaptic Gain Control Drives Sweet and Bitter Taste Integration in Drosophila. Curr. Biol. 24, 1978-1984 (2014).

50. Jeong, Y. T. et al. An Odorant-Binding Protein Required for Suppression of Sweet Taste by Bitter Chemicals. Neuron 79, 725-737 (2013).

51. Lin, S. et al. Neural correlates of water reward in thirsty Drosophila. Nat. Neurosci.17, 1536-1542 (2014). 
52. Breslin, P. A. S., Davidson, T. L. \& Grill, H. J. Conditioned reversal of reactions to normally avoided tastes. Physiol. Behav. 47, 535-538 (1990).

53. Figueroa, J., Gasalla, P., Müller, M. \& Dwyer, D. Socially conditioned flavor preferences with fluids: Transfer with solid foods, palatability, and testing constraints. Physiol. Behav. 223, 112976 (2020).

54. Forestell, C. A. \& LoLordo, V. M. Can Orally Consumed Calories Condition Preferences for Relatively Unacceptable Tastes? Learn. Motiv. 31, 153-179 (2000).

55. Wadhera, D., Capaldi Phillips, E. D. \& Wilkie, L. M. Teaching children to like and eat vegetables. Appetite 93, 75-84 (2015).

56. Trannoy, S., Redt-Clouet, C., Dura, J.-M. \& Preat, T. Parallel Processing of Appetitive Short- and Long-Term Memories In Drosophila. Curr. Biol. 21, 1647-1653 (2011).

57. de Tredern, E. et al. Glial glucose fuels the neuronal pentose phosphate pathway for longterm memory. Cell Rep. 36, 109620 (2021).

58. Dahanukar, A., Lei, Y.-T., Kwon, J. Y.\& Carlson, J. R. Two Gr Genes Underlie Sugar Reception in Drosophila. Neuron 56, 503-516 (2007).

59. Wang, Z., Singhvi, A., Kong, P. \& Scott, K. Taste Representations in the Drosophila Brain. Cell 117, 981-991 (2004).

60. Liu, S., Liu, Q., Tabuchi, M. \& Wu, M. N. Sleep Drive Is Encoded by Neural Plastic Changes in a Dedicated Circuit. Cell 165, 1347-1360 (2016). 
bioRxiv preprint doi: https://doi.org/10.1101/2021.11.12.468444; this version posted November 23, 2021. The copyright holder for this preprint (which was not certified by peer review) is the author/funder, who has granted bioRxiv a license to display the preprint in perpetuity. It is made available under aCC-BY-NC-ND 4.0 International license. 
bioRxiv preprint doi: https://doi.org/10.1101/2021.11.12.468444; this version posted November 23, 2021. The copyright holder for this preprint (which was not certified by peer review) is the author/funder, who has granted bioRxiv a license to display the preprint in perpetuity. It is made available under aCC-BY-NC-ND 4.0 International license.

\section{FIGURES}

A STROBE Memory Assay

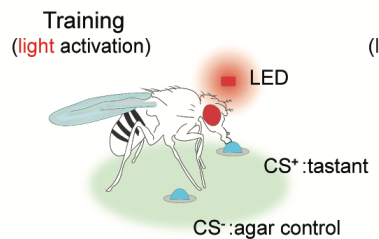

Testing

(light inactivated)

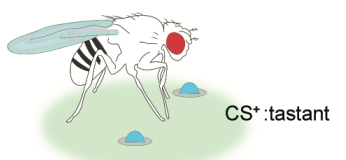

$\mathrm{CS}^{-}$:agar control
B
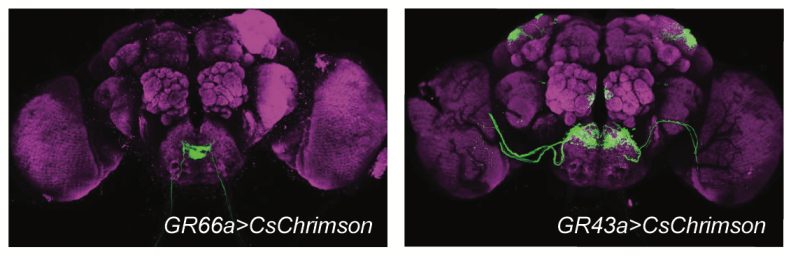

C Aversive Short-Term Memory Assay

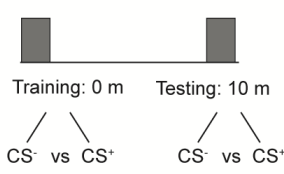

Bitter GRN Activation

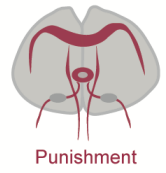

D

Appetitive

Short-Term Memory Assay

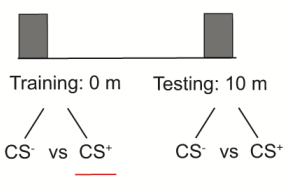

Sweet GRN Activation

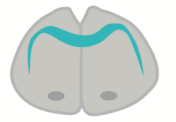

Reward

E

Appetitive

Long-Lasting Memory Assay refeeding: $40 \mathrm{~m}$

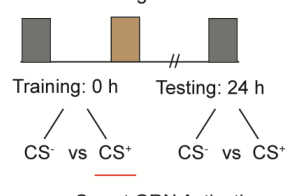

Sweet GRN Activation

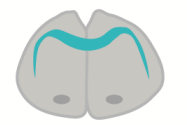

Reward
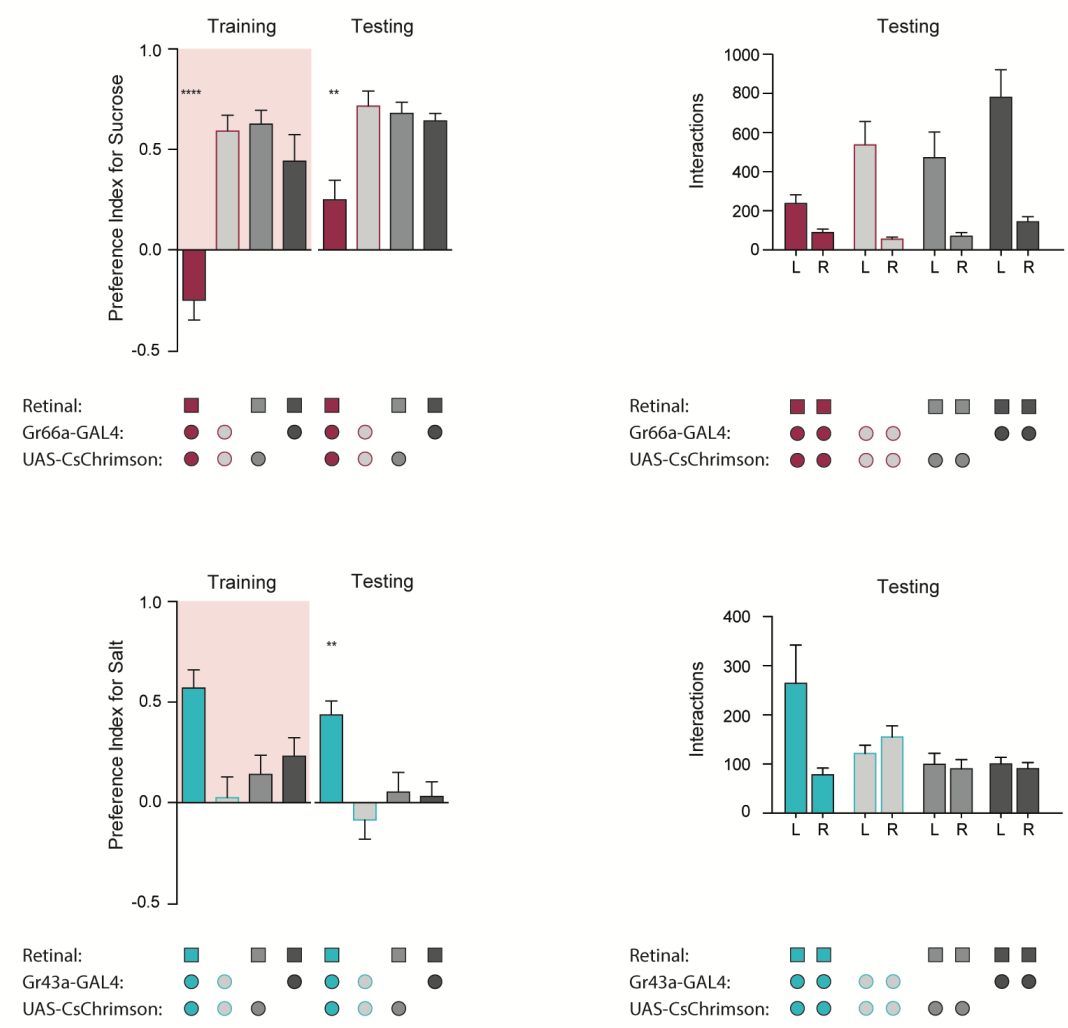

Retinal:

Gr43a-GAL4:

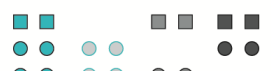

UAS-CsChrimson: 000000
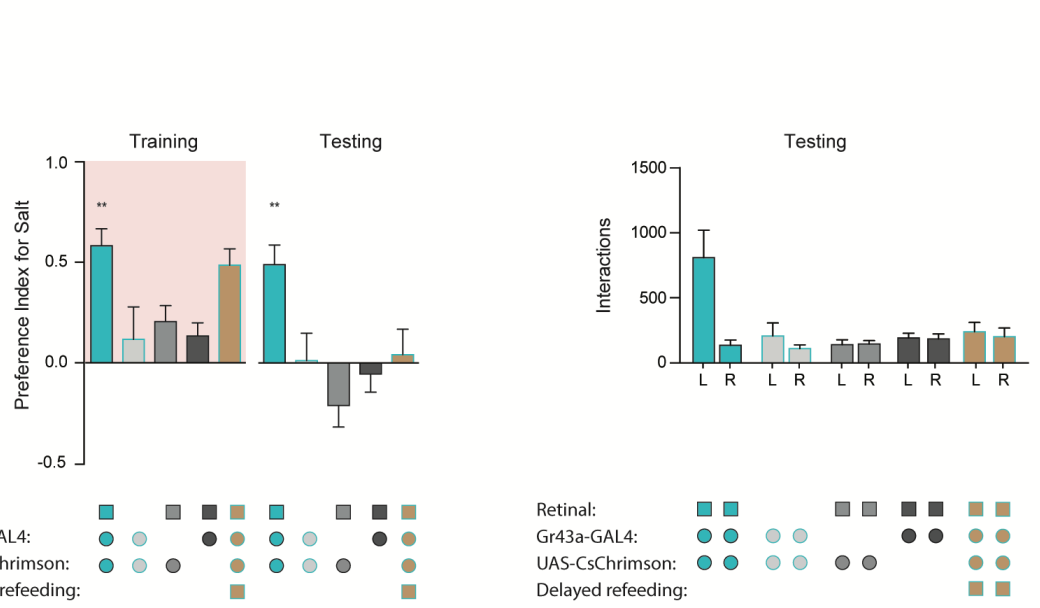

Retinal:

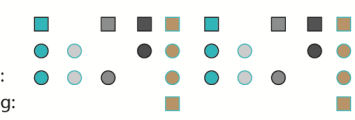

$\begin{array}{lllll}\text { UAS-CsChrimson: } \bigcirc & \circ & 0 & \circ & 0 \\ \text { Delayed refeeding: }\end{array}$
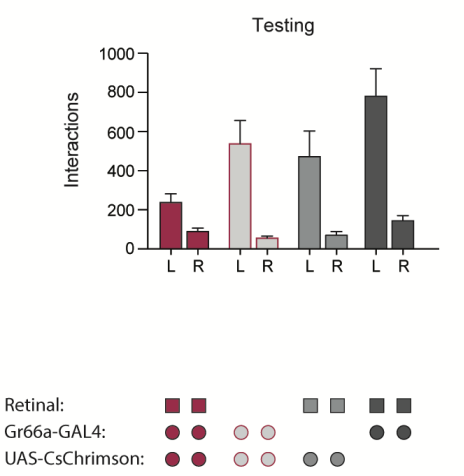

UAS-CsChrimson: 000 


\section{Figure 1: GRNs produce punishment and reward signals capable of facilitating taste} memory formation. (A) Diagram outlining STROBE memory paradigm. Training: starved flies freely interact with a LED-activating tastant for 40 minutes. CsChrimson induces bitter or sweet neuron stimulation upon LED-activation, pairing feeding with punishment or reward. Testing: associative memory is measured by assessing preference for the tastant compared to agar for a 1hour time period. (B) Expression of Gr66a-Gal4 and Gr43a-Gal4 in the fly brain. (C) Schematic of aversive STM timeline and a diagram of activated bitter neurons (left). Preference indices (middle) and tastant interaction numbers (right) for Gr66a>CsChrimson flies compared to genetic controls during training and 10 minutes later upon testing $(n=16-30)$. (D, E) Schematic depicting appetitive short- and long-lasting memory assay timelines, and representative model of sweet GRNs in the SEZ (left). Preference index (middle) and interactions numbers (right) for Gr43a>CsChrimson flies fed all-trans-retinal compared to controls in the short-term $(\mathrm{n}=12-23)$ (D), and long-lasting ( $\mathrm{n}=14-30)$ (E) memory assays (right). Preference index is mean \pm SEM, One-way ANOVA, Dunnett's post hoc test, with: $* * p<0.01, * * * * p<0.0001$. 
A

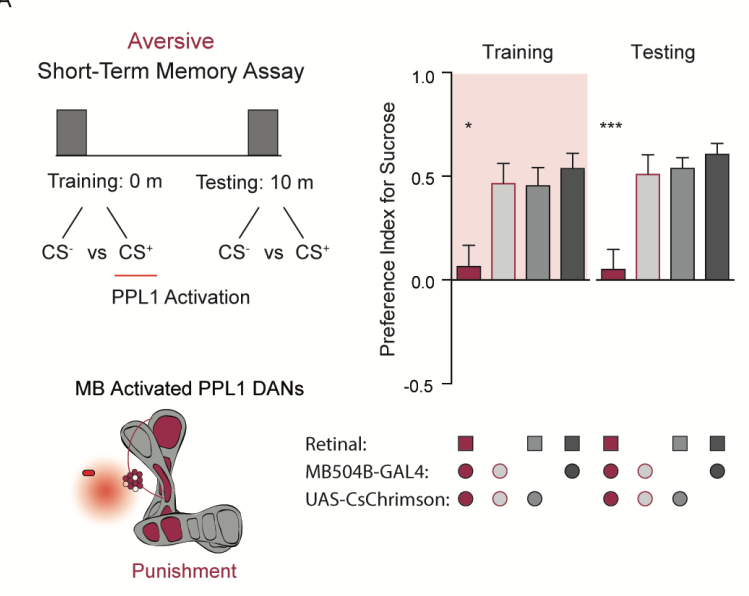

C

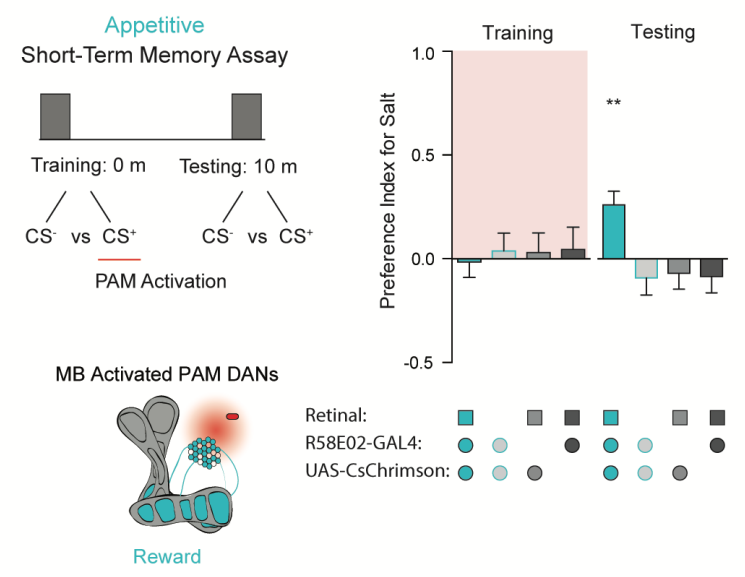

B
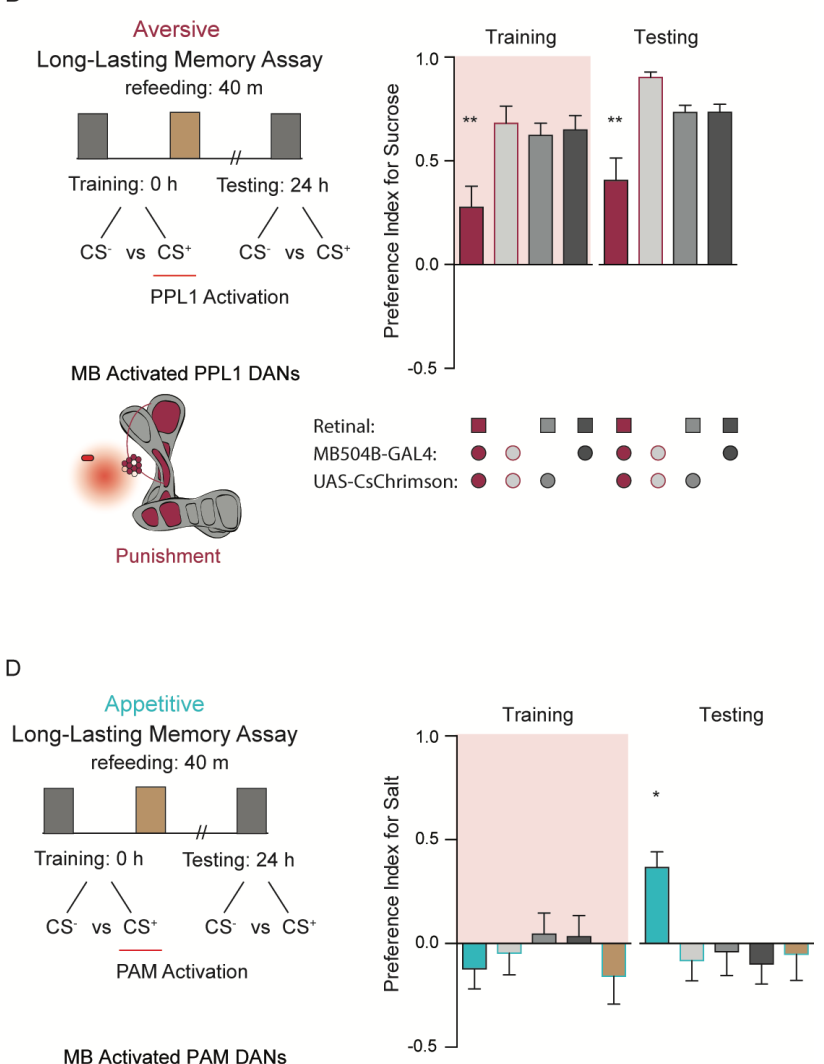

MB Activated PAM DANs

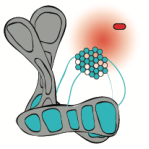

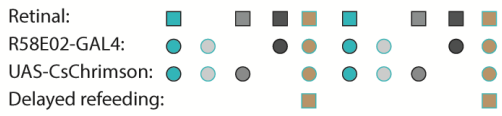

Figure 2: PPL1 and PAM neural activation is sufficient for the induction of short and longlasting taste memories. (A, B) Paradigm timeline schematic and MB model indicating PPL1 compartments activated by optogenetics (left). Preference indices for MB504B>CsChrimson flies and genetic controls in the short-term (n=19-31) (A), and long-lasting ( $n=20-33)(B)$ memory assays (right). (C, D) Assay timeline, and MB model with activated PAM compartments highlighted (left). Preference index for R58E02>CsChrimson flies comparted to controls in the short-term ( $n=25-38)(C)$, and long-lasting ( $n=17-35)$ (D) memory assays (right). Preference indices are mean \pm SEM, One-way ANOVA, Dunnett's post hoc test: $* p<0.05$, ** $p<0.01$, $* * * p<0.001$. 

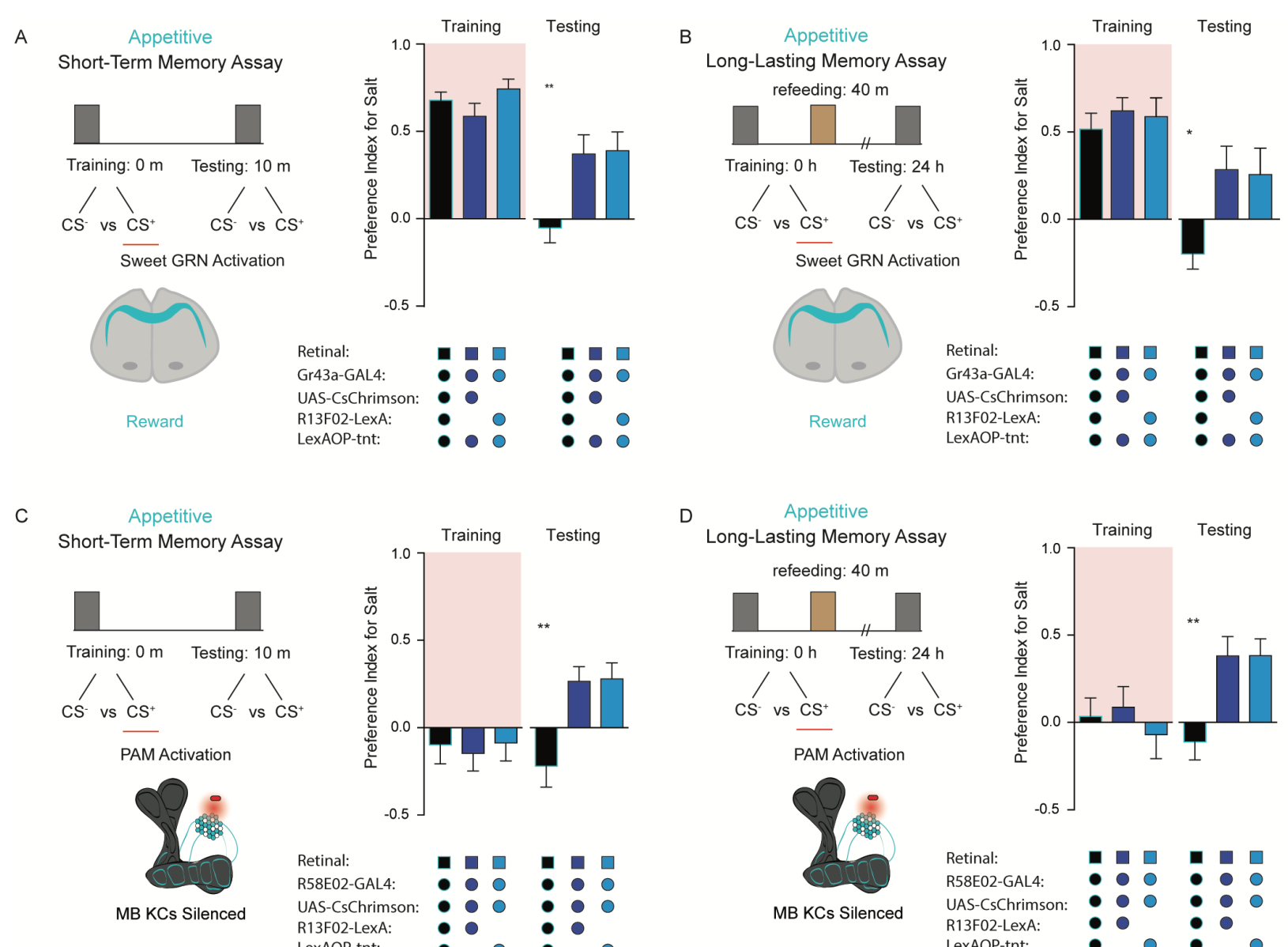

D Appetitive

Long-Lasting Memory Assay
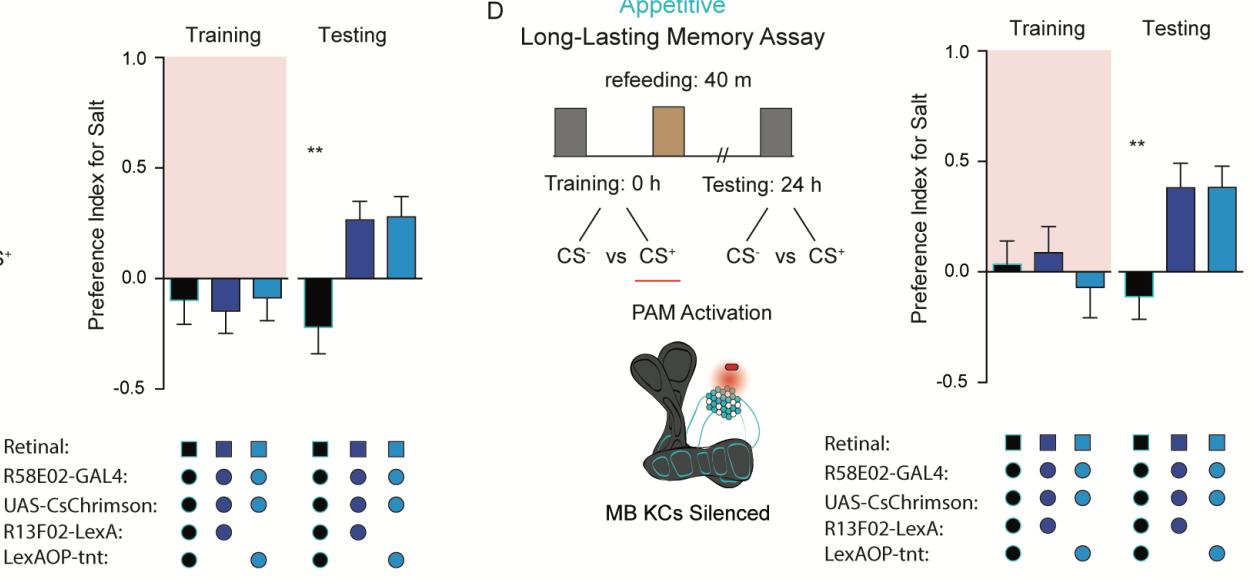

MB KCs Silenced
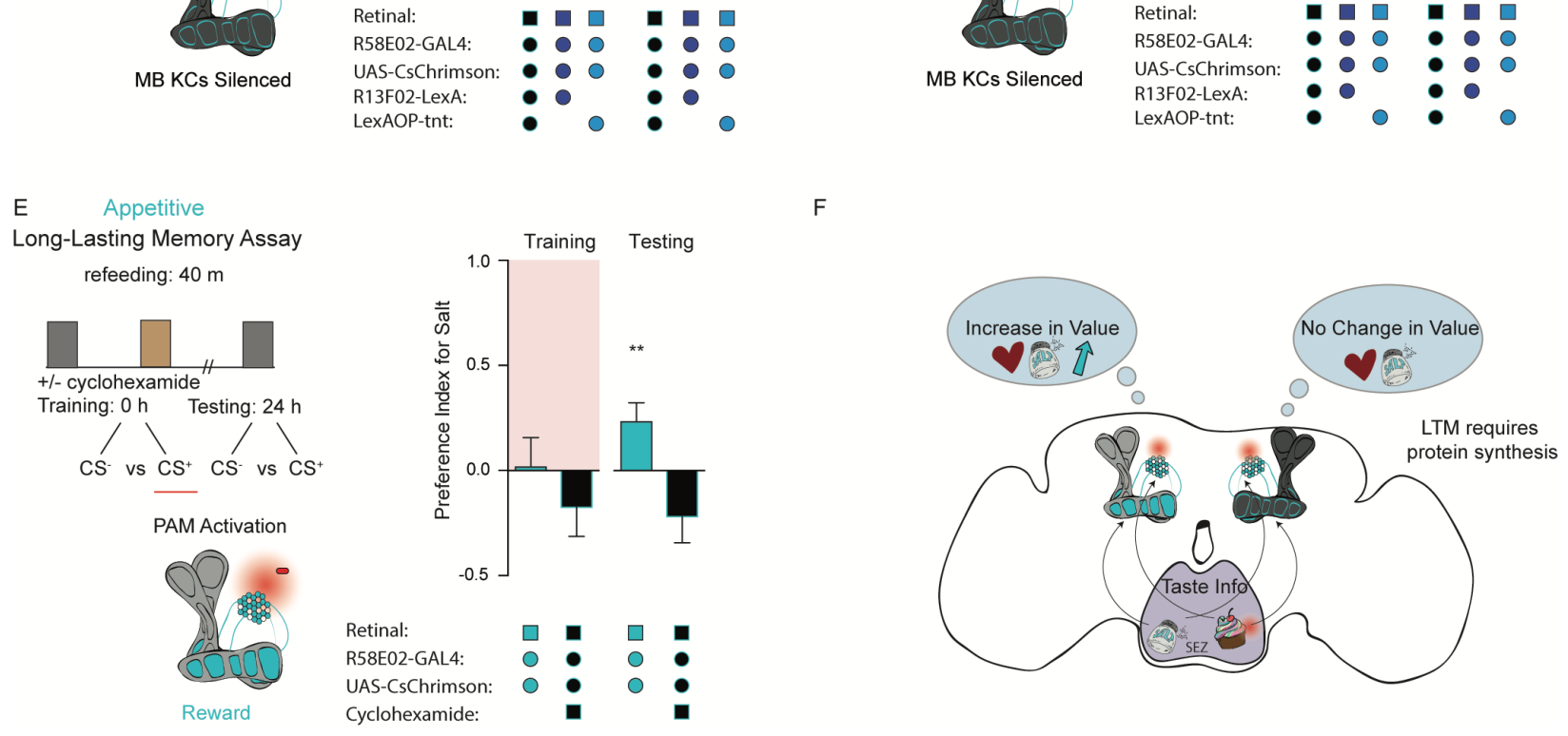

Figure 3: The MB is required for the formation of short- and long-term taste memories (A, B) Preference indices for Gr43a>CsChrimson flies in the short-term (n=16-34) (A) and long-lasting ( $\mathrm{n}=13-27)$ (B) memory assays when the MB is silenced, compared to controls. (C, D) Preference indices for R58E02>CsChrimson flies when the MB is silenced in the short-term $(n=24-28)(C)$ and long-lasting $(n=17-23)(D)$ memory paradigms, compared to controls. $(E)$ 
bioRxiv preprint doi: https://doi.org/10.1101/2021.11.12.468444; this version posted November 23,2021 . The copyright holder for this preprint (which was not certified by peer review) is the author/funder, who has granted bioRxiv a license to display the preprint in perpetuity. It is made available under aCC-BY-NC-ND 4.0 International license.

Preference indices in the long-lasting memory assay for R58E02>CsChrimson flies fed protein synthesis inhibitor cycloheximide compared to vehicle-fed controls ( $\mathrm{n}=17-22)$. (F) Model of appetitive taste memory formation via GRN/PAM activation. Preference indices are mean \pm SEM, $t$-test/One-way ANOVA, Dunnett's post hoc test: ${ }^{*} p<0.05,{ }^{* *} p<0.01$. 
bioRxiv preprint doi: https://doi.org/10.1101/2021.11.12.468444; this version posted November 23, 2021. The copyright holder for this preprint (which was not certified by peer review) is the author/funder, who has granted bioRxiv a license to display the preprint in perpetuity. It is made available under aCC-BY-NC-ND 4.0 International license.
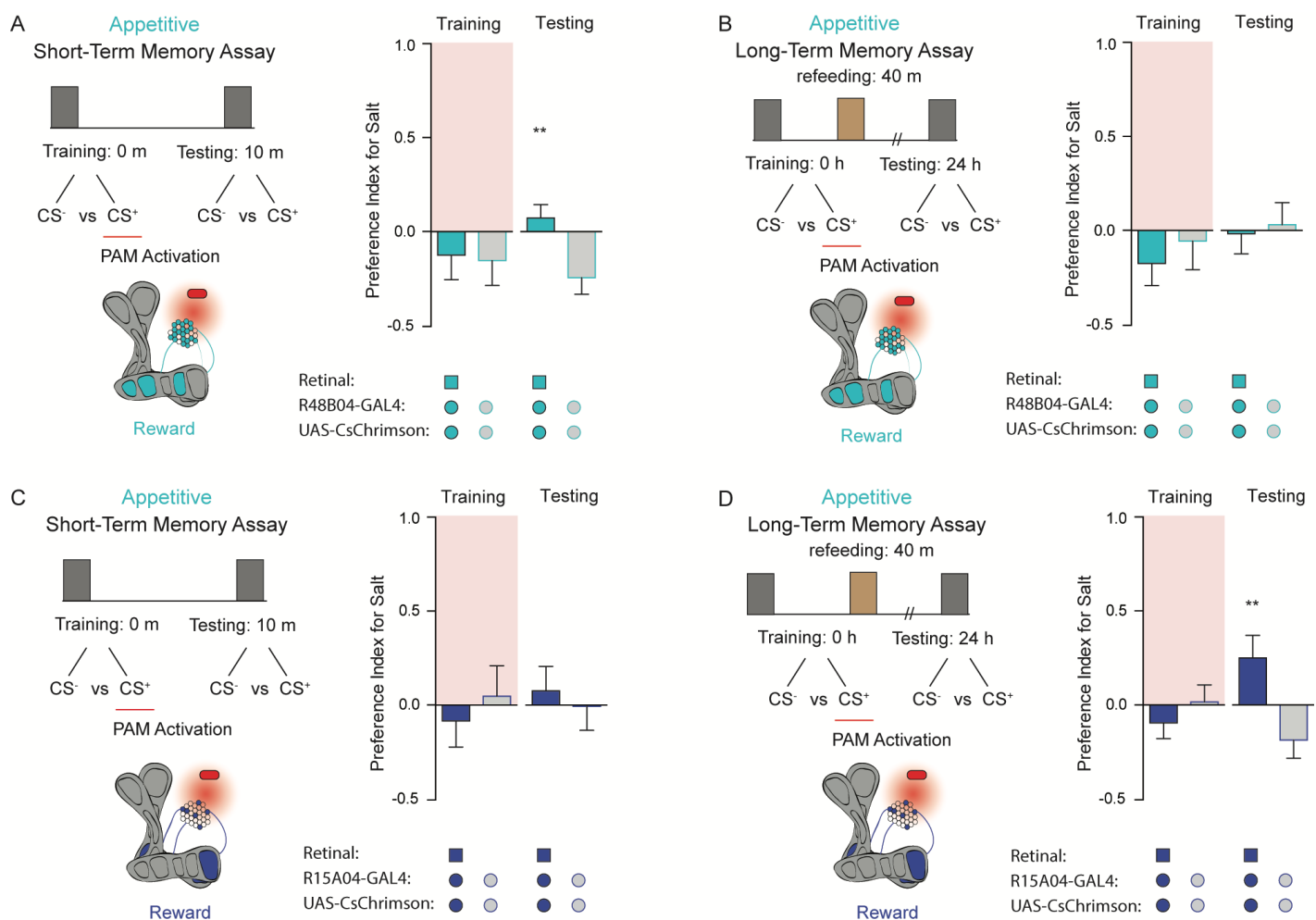

D
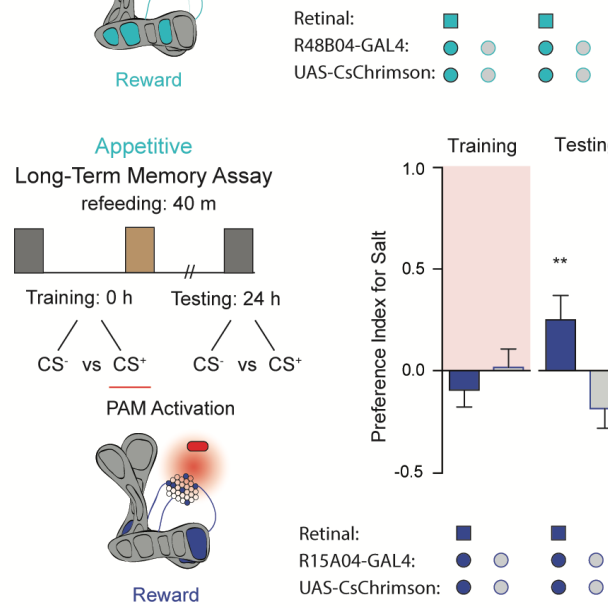

Reward

$\begin{array}{lllll}\text { R15A04-GAL4: } & 0 & 0 & 0 \\ \text { UAS-CsChrimson: } & 0 & \bullet & 0\end{array}$
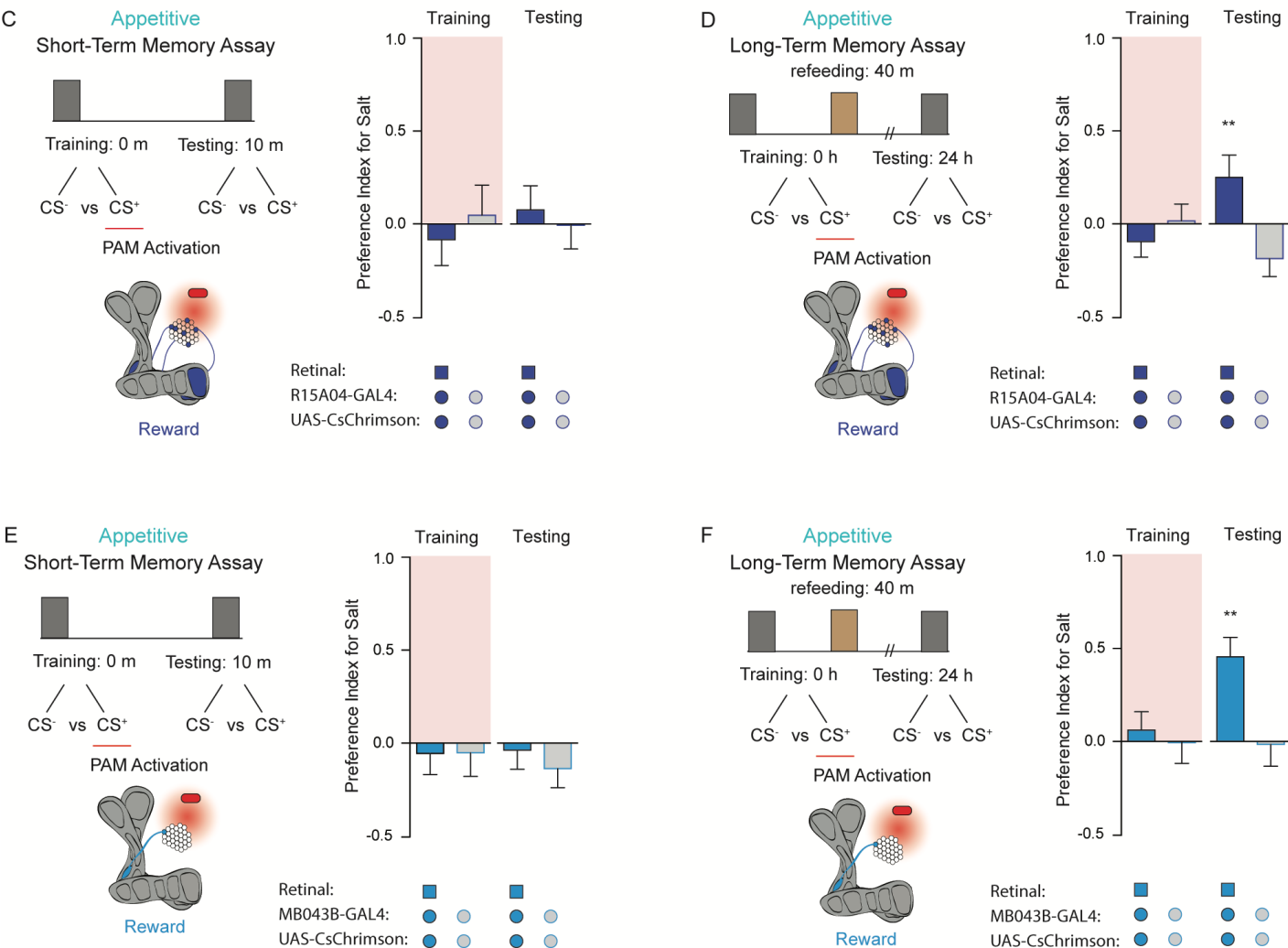

G

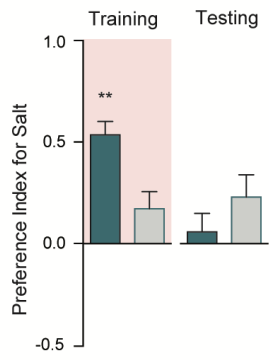

$\mathrm{H}$
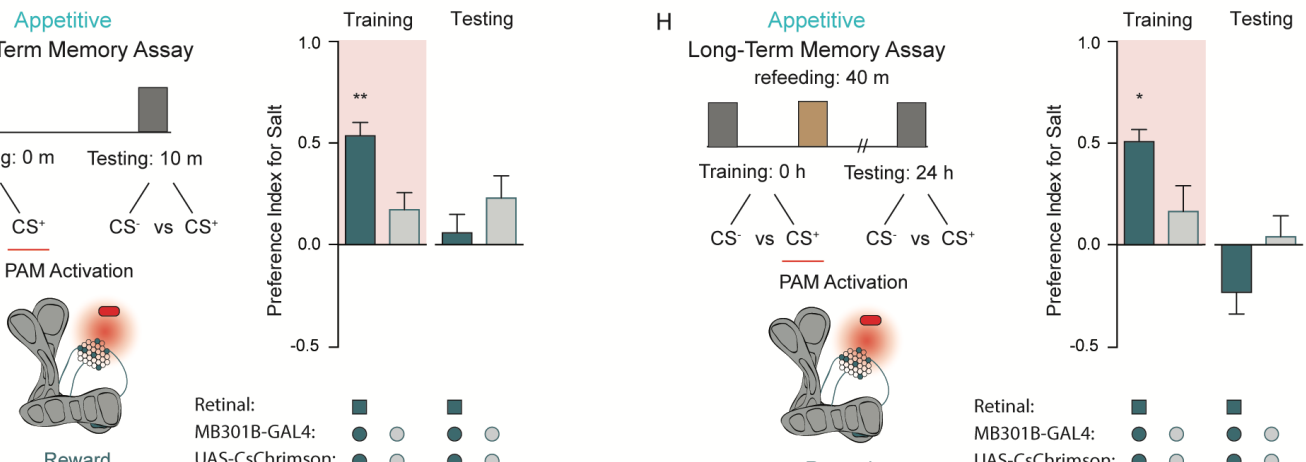

Retinal:

MB301B-GAL4:

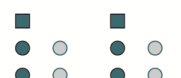

Reward

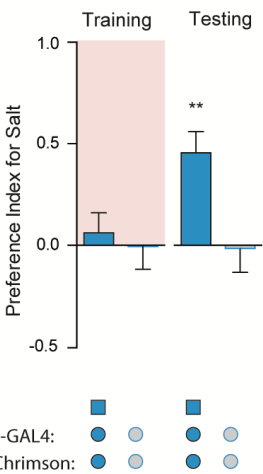

Figure 4: Discrete non-overlapping PAM subpopulations induce appetitive short- and longterm taste memories. (A, B) PAM subpopulation R48B04 innervates highlighted MB 
compartments (left), and preference indices of $R 48 B 04>C s C h r i m s o n$ flies for $75 \mathrm{mM} \mathrm{NaCl}$ is tested in the short-term ( $\mathrm{n}=21-28)(\mathrm{A})$, and long-term $(\mathrm{n}=15-17)(\mathrm{B})$ memory assays with or without retinal. (C, D). PAM subpopulation R15A04 innervates non-overlapping MB subregions compared to R48B04. Preference indices for R15A04>CsChrimson flies fed all-transretinal in the short-term $(n=11-15)(C)$ and long-term $(n=20-27)(D)$ taste memory assays with or without retinal. (E, F) PAM- $\alpha 1$ innervates a single compartment in the MB. Preference indices of $M B 043 B>C s C h r i m s o n$ flies in the short-term $(n=11-14)(\mathrm{E})$ and long-term $(n=19-22)(F)$ memory assays with or without retinal. $(\mathrm{G}, \mathrm{H})$ PAM- $\beta 2 \beta^{\prime} 2 \mathrm{a}$ synapses on the highlighted MB compartment. Preference indices for $M B 301 B>C$ sChrimson flies during the short-term ( $\mathrm{n}=20$ 27) $(\mathrm{G})$ and long-term $(\mathrm{n}=10-15)(\mathrm{H})$ memory assays with or without retinal. Preference indices are mean \pm SEM, $t$-test: $* * p<0.01$. 
A
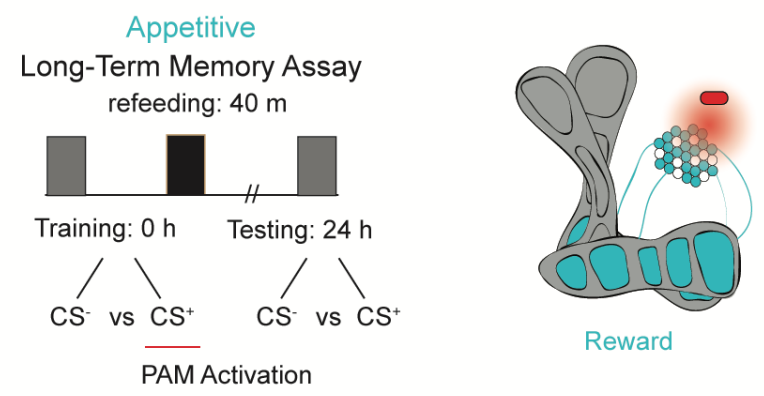

B

Training

Refeeding medium: sucrose L-glucose lactic acid yeast extract L-alanine L-aspartic acid

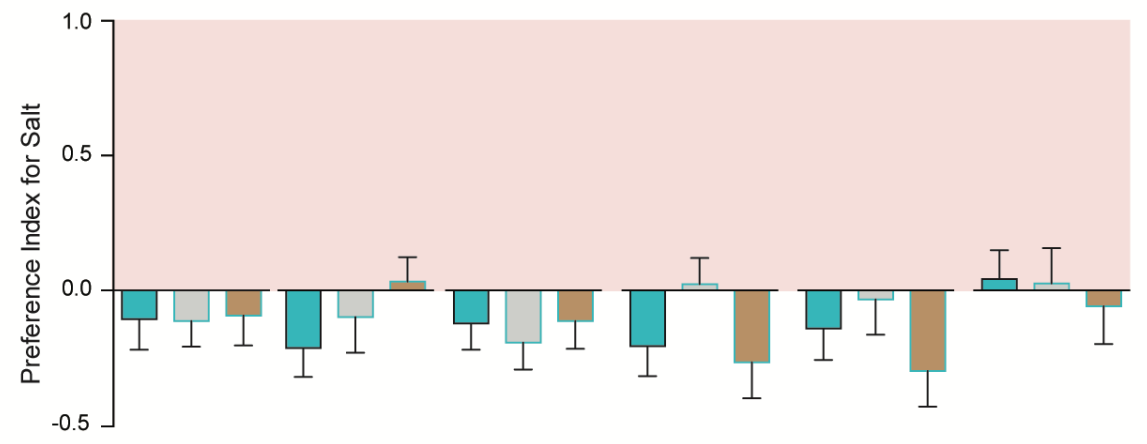

Retinal:

R58E02-GAL4:

UAS-CsChrimson:

Delayed refeeding:

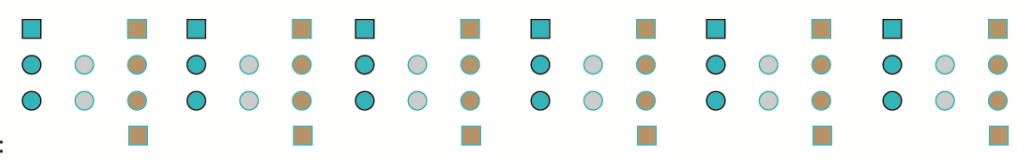

C

Testing

Refeeding medium: sucrose L-glucose lactic acid yeast extract L-alanine L-aspartic acid

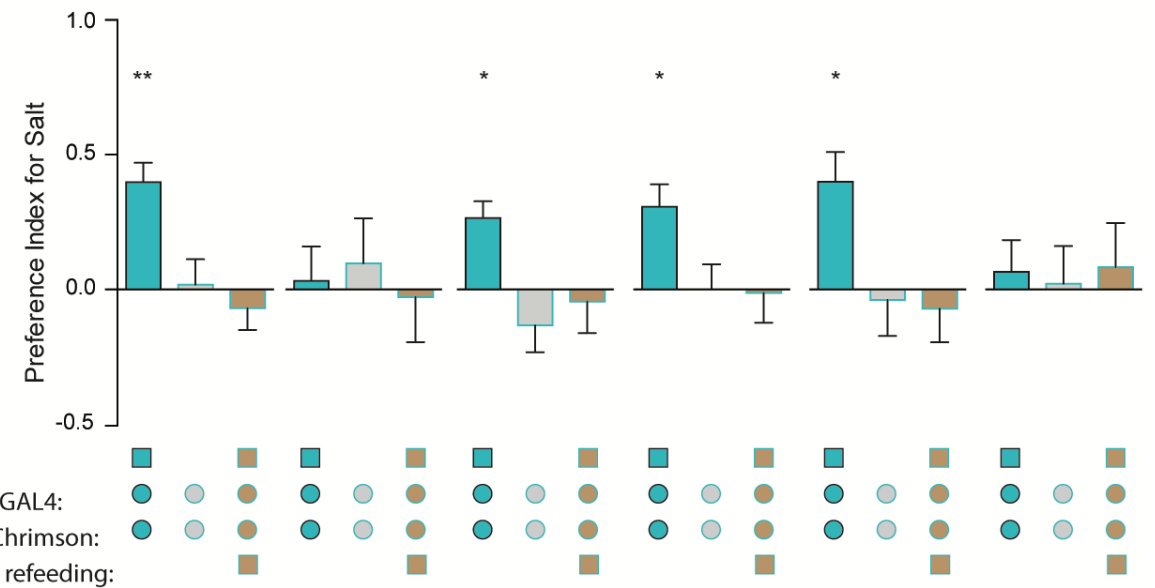

Figure 5: Caloric food sources are required for the formation of associative long-term taste memories. (A) Graphic of the timeline followed for the long-term taste memory paradigm, and the MB compartments innervated by PAM driver R58E02-Gal4. (B, C) Preference indices for 
bioRxiv preprint doi: https://doi.org/10.1101/2021.11.12.468444; this version posted November 23, 2021. The copyright holder for this preprint (which was not certified by peer review) is the author/funder, who has granted bioRxiv a license to display the preprint in perpetuity. It is made available under aCC-BY-NC-ND 4.0 International license.

R58E02>CsChrimson and control flies during training (B), and testing (C), after being refed with a caloric or non-caloric medium $(n=13-28)$. Preference indices are mean \pm SEM, One-way ANOVA, Dunnett's post hoc test: ${ }^{*} p<0.05, * * p<0.01$. 
A

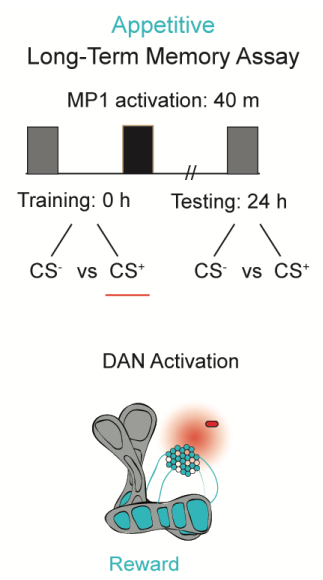

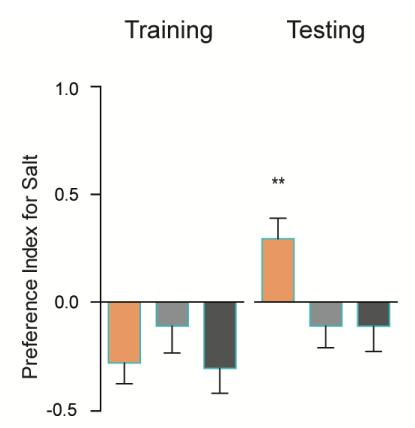

C

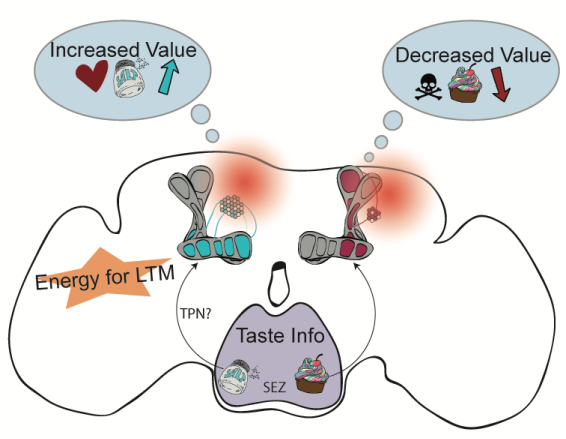

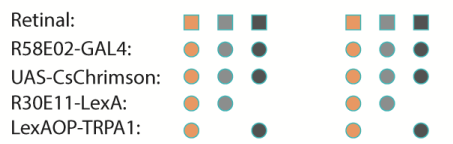

Figure 6: MB-MP1 neuron activation post-training replaces energy signal required for the formation of LTM. (A) Graphic of timeline followed for the LTM taste assay. Preference indices during training and testing for R58E02>CsChrimson flies fed all-trans-retinal, with MBMP1 neurons thermogenetically activated post training using R30E11>TRPA1, compared to controls without MB-MP1 activation (n=18-29). (C) Schematic depicting pathway of activation. Preference indices are mean \pm SEM, One-way ANOVA, Dunnett's post hoc test: $* * p<0.01$. 